\title{
Efficacy of the Self-management Support System DialBetesPlus for Diabetic Kidney Disease: Protocol for a Randomized Controlled Trial
}

Yuki Kawai ${ }^{1,2^{*}}$, MD; Akiko Sankoda ${ }^{1 *}$, MD, PhD; Kayo Waki ${ }^{1,3}$, MD, MPH, PhD; Kana Miyake ${ }^{1,3}$, MD, PhD; Aki Hayashi $^{1}$, MD, PhD; Makiko Mieno ${ }^{4}$, MHS, PhD; Hiromichi Wakui ${ }^{2}, \mathrm{MD}, \mathrm{PhD}$; Yuya Tsurutani ${ }^{5}$, MD, PhD; Jun Saito $^{5}$, MD, PhD; Nobuhito Hirawa ${ }^{6}, \mathrm{MD}, \mathrm{PhD}$; Tadashi Yamakawa ${ }^{7}, \mathrm{MD}$, PhD; Shiro Komiya ${ }^{2}, \mathrm{MD}$; Akihiro Isogawa ${ }^{8}, \mathrm{MD}, \mathrm{PhD}$; Shinobu Satoh ${ }^{9}, \mathrm{MD}, \mathrm{PhD}$; Taichi Minami ${ }^{10}, \mathrm{MD}, \mathrm{PhD}$; Uru Osada ${ }^{10}, \mathrm{MD}, \mathrm{PhD}$; Tamio Iwamoto ${ }^{11}$, MD, PhD; Tatsuro Takano ${ }^{12}$, MD, PhD; Yasuo Terauchi ${ }^{13}, \mathrm{MD}, \mathrm{PhD}$; Kouichi Tamura ${ }^{2}$, MD, PhD; Toshimasa Yamauchi $^{3}$, MD, PhD; Takashi Kadowaki ${ }^{14,15}$, MD, PhD; Masaomi Nangaku ${ }^{16}$, MD, PhD; Naoki Kashihara ${ }^{17}$, MD, $\mathrm{PhD}$; Kazuhiko Ohe ${ }^{1}, \mathrm{MD}, \mathrm{PhD}$

\footnotetext{
${ }^{1}$ Department of Planning, Information and Management, University of Tokyo Hospital, Tokyo, Japan

${ }^{2}$ Department of Medical Science and Cardiorenal Medicine, Yokohama City University Graduate School of Medicine, Yokohama, Japan

${ }^{3}$ Department of Diabetes and Metabolic Diseases, Graduate School of Medicine, The University of Tokyo, Tokyo, Japan

${ }^{4}$ Department of Medical Informatics, Center for Information, Jichi Medical University, Shimotsuke, Japan

${ }^{5}$ Endocrinology and Diabetes Center, Yokohama Rosai Hospital, Yokohama, Japan

${ }^{6}$ Department of Nephrology and Hypertension, Yokohama City University Medical Center, Yokohama, Japan

${ }^{7}$ Department of Endocrinology and Diabetes, Yokohama City University Medical Center, Yokohama, Japan

${ }^{8}$ Division of Diabetes, Mitsui Memorial Hospital, Tokyo, Japan

${ }^{9}$ Department of Endocrinology and Metabolism, Chigasaki Municipal Hospital, Chigasaki, Japan

${ }^{10}$ Department of Diabetes and Endocrinology, Saiseikai Yokohamashi Nanbu Hospital, Yokohama, Japan

${ }^{11}$ Department of Nephrology and Hypertension, Saiseikai Yokohamashi Nanbu Hospital, Yokohama, Japan

${ }^{12}$ Department of Diabetes and Endocrinology, Fujisawa City Hospital, Fujisawa, Japan

${ }^{13}$ Department of Endocrinology and Metabolism, Yokohama City University Graduate School of Medicine, Yokohama, Japan

${ }^{14}$ Department of Prevention of Diabetes and Lifestyle-Related Diseases, Graduate School of Medicine, The University of Tokyo, Tokyo, Japan

${ }^{15}$ Toranomon Hospital, Tokyo, Japan

${ }^{16}$ Division of Nephrology and Endocrinology, Graduate School of Medicine, The University of Tokyo, Tokyo, Japan

${ }^{17}$ Department of Nephrology and Hypertension, Kawasaki Medical School, Kurashiki, Japan

*these authors contributed equally
}

\section{Corresponding Author:}

Kayo Waki, MD, MPH, PhD

Department of Planning, Information and Management, University of Tokyo Hospital

7-3-1 Hongo, Bunkyo-ku

Tokyo, 113-8655

Japan

Phone: 81358009129

Fax: 81358009129

Email: kwaki-tky@m.u-tokyo.ac.jp

\section{Abstract}

Background: Diabetic kidney disease (DKD) is one of the main complications of type 2 diabetes mellitus (T2DM). DKD is a known risk factor for end-stage renal disease, cardiovascular disease, and all-cause death. Effective intervention for early-stage DKD is vital to slowing down the progression of kidney disease and improve prognoses. Mobile health (mHealth) is reportedly effective in supporting patients' self-care and improving glycemic control, but the impact of mHealth on DKD has yet to be shown. 
Objective: The purpose of this study is to evaluate the efficacy of standard therapy with the addition of a self-management support system, DialBetesPlus, in patients with DKD and microalbuminuria.

Methods: This study is a prospective, randomized, open-label, multicenter clinical trial. The target population consists of 160 patients diagnosed with T2DM accompanied by microalbuminuria. We randomly assigned the patients to 2 groups-the intervention group using DialBetesPlus in addition to conventional therapy and the control group using conventional therapy alone. DialBetesPlus is a smartphone application that supports patients' self-management of T2DM. The study period was 12 months, with a follow-up survey at 18 months. The primary outcome was a change in albuminuria levels at 12 months. Secondary outcomes included changes in physical parameters, blood test results (glycemic control, renal function, and lipid metabolism), lifestyle habits, self-management scores, medication therapy, and quality of life.

Results: The study was approved in April 2018. We began recruiting patients in July 2018 and completed recruiting in August 2019. The final 18-month follow-up was conducted in March 2021. We recruited 159 patients and randomly allocated 70 into the intervention group and 61 into the control group, with 28 exclusions due to withdrawal of consent, refusal to continue, or ineligibility. The first results are expected to be available in 2021.

Conclusions: This is the first randomized controlled trial assessing the efficacy of mHealth on early-stage DKD. We expect that albuminuria levels will decrease significantly in the intervention group due to improved glycemic control with ameliorated self-care behaviors.

Trial Registration: UMIN-CTR UMIN000033261; https://upload.umin.ac.jp/cgi-open-bin/ctr/ctr_view.cgi?recptno=R000037924 International Registered Report Identifier (IRRID)： DERR1-10.2196/31061

(JMIR Res Protoc 2021;10(8):e31061) doi: 10.2196/31061

\section{KEYWORDS}

diabetic kidney disease; microalbuminuria; albuminuria; diabetes mellitus; self-management support system; mHealth; randomized controlled trial; diabetes; kidney; chronic disease; support; self-management; efficacy; protocol; therapy; intervention; self-care; behavior

\section{Introduction}

The increase in type 2 diabetes mellitus (T2DM) worldwide is the primary cause of the increasing prevalence of end-stage renal disease (ESRD) [1]. The number of ESRD patients under renal replacement therapy is estimated to be more than 3 million worldwide and is projected to increase to more than 5 million people by 2030 [2]. Therefore, effective management of diabetic kidney disease (DKD) is essential, especially in the early stages. It has been demonstrated that albuminuria is one of the earliest detectible clinical manifestations of kidney disease and is a potent risk factor for ESRD and all-cause death $[3,4]$. Renin-angiotensin blockades are approved as a strategy to reduce urinary albumin excretion and are in widespread use [5,6]. Recently, glucagon-like peptide-1 analogs and sodium-glucose cotransporter-2 inhibitors have also been shown to slow the progression of DKD potentially $[7,8]$. Despite all these pharmacological interventions, the overall prevalence of DKD did not change significantly among US adults with diabetes from 1988 to 2014 [9]. Moreover, with 415 million patients with diabetes reported worldwide in 2015 , the number is still increasing and is predicted to rise to 642 million by 2040 [10]. There is an increasingly urgent need for a more effective methodology to prevent the progression of DKD.

Modification of dietary and exercise habits is still one of the most fundamental therapeutic strategies. However, a sufficient support system for patients' self-care has not been established. Whereas mobile health (mHealth) interventions supporting patients' self-management have shown effectiveness in glycemic control of T2DM [11-14], the effects of mHealth on DKD have yet to be evaluated.
In 2015, over half of the people with diabetes worldwide lived in Southeast Asia or the Western Pacific Region. In Asia, it is estimated that the number of patients with diabetes will increase 1.8 times by 2040 , and ESRD prevalence will rise sharply relative to other regions [2,10]. However, a population-based approach is also reported to decrease diabetes-related ESRD successfully $[15,16]$. mHealth might be a promising population-based approach, providing basic treatment and reliable information with easy accessibility at low cost.

We previously reported that the self-management support system DialBetics significantly improved glycemic control in T2DM patients, possibly due to improvement in diet and exercise $[17,18]$. We have since developed an updated system, DialBetesPlus, to investigate the effect of mHealth on DKD. In this study, we investigate the impact of DialBetesPlus on patients with DKD and microalbuminuria.

\section{Methods}

\section{Study Design}

This study is a prospective, randomized, open-label, multicenter clinical trial. The study was conducted at 8 hospitals located in Tokyo and Kanagawa, Japan (Textbox 1). We aimed to evaluate the efficacy of DialBetesPlus on microalbuminuria in T2DM patients. Figure 1 shows a flowchart of this trial. The intervention group used DialBetesPlus and conventional therapy for 12 months, while the control group was treated with conventional therapy alone. The primary outcome was a change in albuminuria levels in a first-morning void after 12 months. The final follow-up was in 18 months, 6 months after the 12-month intervention period. 
Textbox 1. List of trial institutions.

- The University of Tokyo Hospital

- Yokohama City University Hospital

- Yokohama City University Medical Center

- Yokohama Rosai Hospital

- Saiseikai Yokohamashi Nanbu Hospital

- Fujisawa City Hospital

- Chigasaki Municipal Hospital

- Mitsui Memorial Hospital

Figure 1. Protocol flowchart.

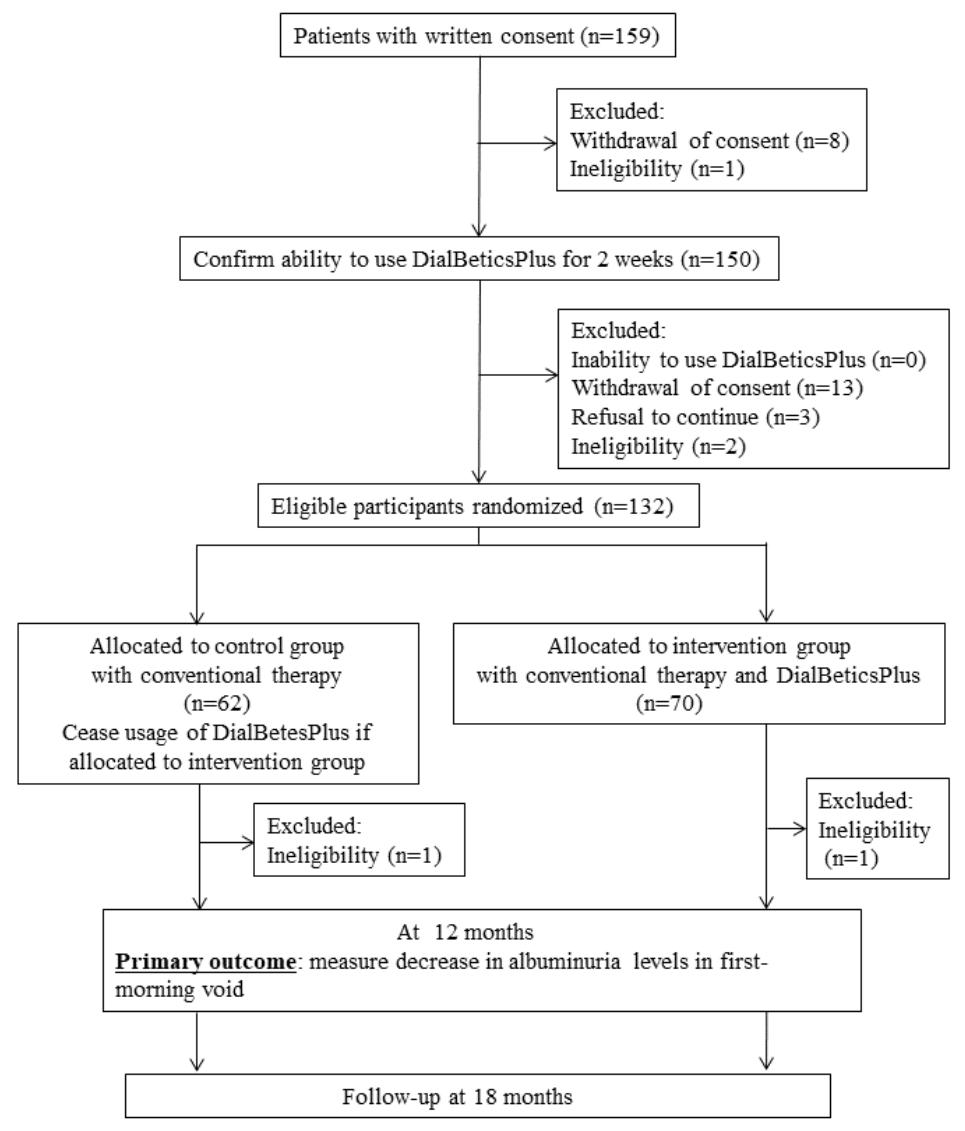

\section{Participants}

We recruited patients who passed all the inclusion and exclusion criteria before randomization (Textbox 2). The criteria were designed to include T2DM patients experiencing the early stage of DKD without any restrictions on physical activity. Eligible participants had T2DM with microalbuminuria (30-299 mg/g creatinine), hemoglobin $\mathrm{A}_{1 \mathrm{c}}\left(\mathrm{HbA}_{1 \mathrm{c}}\right)$ of $6.5 \%$ or more, and an estimated glomerular filtration rate (eGFR) of $45 \mathrm{~mL} / \mathrm{min} / 1.73 \mathrm{~m}^{2}$ or more. We obtained written, informed consent from all the patients participating in this study. Prior to the randomization, all enrolled patients were asked to use DialBetesPlus for 2 weeks to confirm their ability to use the system and devices properly. Eligible participants were those able to use DialBetesPlus for over 7 days during the initial 2-week confirmation period. The participants were randomized into either the intervention or control group in a one-to-one fashion based on albuminuria levels, gender, and age. The research team consisted of diabetologists, nephrologists, pharmacists, dieticians, nurses, a laboratory technician, and technical support experts. While the team handled technical troubles and monitored usage of the system, the patients continued to consult their attending doctors about their general health status. If 7 days passed without any input into the system, an alert was sent to encourage the patient to resume providing input. If 3 weeks passed without a patient inputting any data, we defined the patient as a dropout. Participants with whom the study team has lost contact were also treated as dropouts. The criteria for study discontinuance were a serious adverse event, patient request for discontinuance, pregnancy, or the judgment of a lead physician. 
Textbox 2. Inclusion and exclusion criteria.

\section{Inclusion criteria}

- $\quad$ Diagnosed with T2DM

- $\quad \mathrm{HbA}_{1 \mathrm{c}} 6.5 \%$ or more

- $\quad$ Between 20 and 75 years of age

- $\quad$ BP lower than $180 / 110 \mathrm{mmHg}$

- $\quad$ eGFR $45 \mathrm{~mL} / \mathrm{min} / 1.73 \mathrm{~m}^{2}$ or more

- Two detected instances of microalbuminuria (30-299 mg/g creatinine) in spot urine samples prior to study enrollment

- $\quad$ BMI of $22 \mathrm{~kg} / \mathrm{m}^{2}$ or more

- No history of severe hypoglycemia requiring additional medical support

- No history of the following symptoms indicating hypoglycemia within the last 3 months: palpitations, tremors, dizziness, anxiety, loss of consciousness, sweating, facial pallor, tachycardia, headache, sleepiness, blurred vision, or convulsions

- Regular patients of hospitals listed in Textbox 1

- $\quad$ Signatories of the informed consent form

\section{Exclusion criteria}

- Use of cardiac pacemaker

- Hyperthyroidism diagnosis, under medication other than thyroid hormone supplementation in the last year

- Medical instability or exercise restriction as ordered by a physician, with autoimmune, heart, liver, digestive, neurological, or respiratory disease

- $\quad \mathrm{Hb}$ less than $10 \mathrm{~g} / \mathrm{dL}$

- Albumin $3.0 \mathrm{~g} / \mathrm{dL}$ or less

- $\quad$ eGFR less than $45 \mathrm{~mL} / \mathrm{min} / 1.73 \mathrm{~m}^{2}$

- Those with preproliferative diabetic retinopathy within one year of signing consent forms

- Inability to exercise

- Pregnancy, potential planned pregnancy, or lactating

- Participation in other clinical trials

- Under a diet that restricts protein

- Judged as ineligible by doctor's discretion for other reasons

\section{Design of DialBetesPlus}

The details of the DialBetesPlus system are shown in Figure 2. Patients measured blood glucose, blood pressure (BP), body weight, and pedometer counts at home. The data were transferred from each device to a smartphone by either Near Field Communication (NFC) or Bluetooth, and then immediately sent to a server, where they were automatically evaluated following the Japan Diabetes Society (JDS) guideline's target values [19]. Optimal values include blood glucose below 110 $\mathrm{mg} / \mathrm{dl}$ before breakfast and $140 \mathrm{mg} / \mathrm{dl}$ at bedtime, BP below $125 / 75 \mathrm{mmHg}$, and pedometer counts above 8000 steps per day.
DialBetesPlus determined if each reading satisfies the JDS guideline requirements and immediately sent the results to the patient's smartphone. Patients also entered the contents and quantity of their meals and the type and duration of exercise not counted by a pedometer. Then, their intake and consumed calories were automatically calculated and sent to the smartphone along with specific advice regarding lifestyle modifications based on JDS guidelines. Critical values with blood glucose levels above $400 \mathrm{mg} / \mathrm{dl}$ or below $70 \mathrm{mg} / \mathrm{dl}$, systolic BP above $220 \mathrm{mmHg}$, or diastolic BP above $110 \mathrm{mg}$ were automatically reported to the research team, and the team informed attending doctors when necessary. 
Figure 2. DialBetesPlus overview. NFC: near field communication.

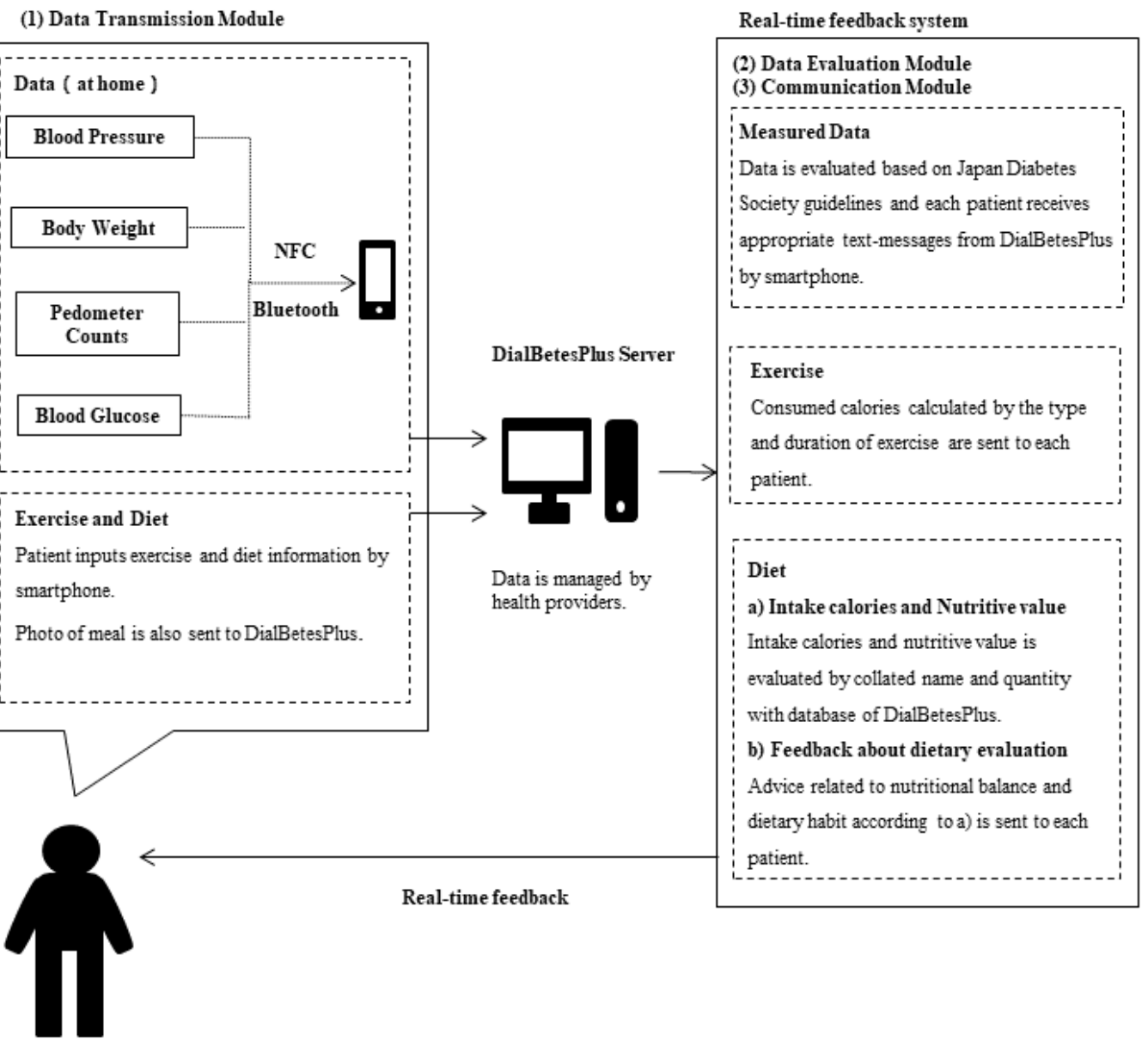

\section{The DialBetesPlus Intervention and Control}

The patients allocated to the intervention group used DialBetesPlus for 12 months. Patients in the intervention group receive an NFC-enabled glucometer (MS-FR201B; Terumo), a Bluetooth-enabled BP monitor (HEM-7271T; Omron), a pedometer (MT-KT02DZ; Terumo), and a scale (HBF-255T; Omron). These devices were all paired with a single provided smartphone (Arrows F-02H; Fujitsu or Galaxy Note3 SC-01F; Samsung) that transmits readings to the DialBetesPlus server via a wireless network. In addition, as a part of standard therapy, the participants in the control group were provided with sphygmomanometers to measure $\mathrm{BP}$ at home.

In this study, we divided the participants into 2 models (a hospital-led model and a pharmacy-led model) depending on the location of study enrollment. In the hospital-led model (the University of Tokyo Hospital, Yokohama City University Hospital, Saiseikai Yokohamashi Nanbu Hospital, and Fujisawa City Hospital), the instructions included guidance on DialBetesPlus use provided by health care providers at the patient's hospital. In a pharmacy-led model (run by the other 4 hospitals), instructions on using DialBetesPlus were provided by pharmacists supporting the study. The pharmacists are Nihon Chouzai Co, Ltd employees, with whom we signed a business cooperation contract to conduct the pharmacy-led model portion of the study.

\section{Sample Size}

The primary outcome of this study is a change in albuminuria levels at 12 months. We estimated that baseline first-morning-void albuminuria level would be 200 plus or minus $200 \mathrm{mg} / \mathrm{g}$ creatinine and hypothesized the difference in the change in albuminuria between the intervention group and the control group at 12 months would be 100 plus or minus 200 $\mathrm{mg} / \mathrm{g}$ creatinine. Based on previous reports [20,21], we calculated 63 patients were required in each group to achieve a 2-sided significance level of .05 and a statistical power of $80 \%$. Factoring ineligibility and dropouts, we calculated a final target number of 80 patients per group.

\section{Study Outcomes}

We defined the primary outcome as a change in albuminuria levels in the first-morning void over 12 months. A first-morning void is less influenced by hydration and physical activity than a spot urine sample [22-25]. As the participants in this study are restricted to patients with microalbuminuria but without macroalbuminuria, we validated albuminuria in a first-morning void.

The secondary outcomes were changes in physical parameters (BMI and BP), blood tests, lifestyle habits, self-management, medication therapy, adherence to the diabetes medication regimen, and quality of life $(\mathrm{QoL})$. Blood test parameters included renal function (eGFR), glycemic control ( $\mathrm{HbA}_{1 \mathrm{c}}$ and fasting blood glucose), and lipid metabolism (high-density 
lipoprotein cholesterol [HDL-C], low-density lipoprotein cholesterol [LDL], and triglycerides [TG]). We also assessed all-cause deaths, composite cardiovascular outcomes, composite renal endpoints, safety, and the usage frequency of DialBetesPlus.

Changes in lifestyle habits were assessed via food-log diaries covering 3 days. Dietitians calculated caloric intake and nutrient balance. The Summary of Diabetes Self-Care Activities Measure (SDSCA)—which evaluates 7 aspects of the diabetes regimen: general diet, specific diet, exercise, medication taking, blood-glucose testing, foot care, and smoking — is a widely used self-reporting tool for patients with diabetes in the United States [26]. We evaluated changes in self-management using the Japanese version of the SDSCA, the J-SDSCA. Change in medication therapy was assessed by evaluating prescriptions. Diabetes medication adherence was monitored using the 8-item Morisky Adherence Scale (MMAS-8). The MMAS-8 is reliable and valid in measuring the adherence of patients with multiple chronic conditions worldwide, including T2DM [27-29]. We measured patients' QoL using the Japanese version of the Audit of Diabetes-Dependent Quality of Life (JP-ADDQoL). The ADDQoL is a diabetes-specific QoL measurement scale producing reliable and valid scores [30,31]. JP-ADDQoL also showed adequate reliability and acceptable validity [32].
Composite cardiovascular outcomes included the first occurrence and recurrence of myocardial infarction and stroke, the first occurrence of percutaneous coronary intervention and coronary artery bypass, hospitalization for unstable angina and heart failure, and cardiovascular death. The composite renal endpoint was defined as ESRD and more than a 2-fold increase in serum creatinine.

To ensure safety, we monitored the number of hypoglycemic events, other adverse events, and issues with the DialBetesPlus system throughout the study. The results of the hospital-led model and the pharmacy-led model will also be compared.

\section{Data Collection}

At registration, we collected information on patients' background, albuminuria level in a first-morning void, physical parameters, medications, blood tests, food-log diaries, J-SDSCA, MMAS-8, and JP-ADDQoL. Follow-up visits were scheduled at 2 months (plus or minus 4 weeks), 6 months (plus or minus 6 weeks), 12 months (plus or minus 6 weeks), and 18 months (plus or minus 6 weeks). We collected and recorded information according to the schedule in Table 1. Even if the interventional treatment was discontinued, we collected as much information as possible with the consent of participants.

Table 1. Assessment and follow-up schedule.

\begin{tabular}{|c|c|c|c|c|c|}
\hline \multirow[t]{2}{*}{ Assessments } & \multicolumn{5}{|c|}{ Follow-up period } \\
\hline & Registration & 2 months & 6 months & 12 months & 18 months \\
\hline \multicolumn{6}{|l|}{ Patient background } \\
\hline \multicolumn{6}{|c|}{ Albuminuria level in a first-morning void } \\
\hline \multicolumn{6}{|l|}{ Albuminuria level in } \\
\hline \multicolumn{6}{|l|}{ Physical parameters } \\
\hline \multicolumn{6}{|l|}{ Medication therapy } \\
\hline \multicolumn{6}{|l|}{ Blood test } \\
\hline \multicolumn{6}{|l|}{ Dietary intake log } \\
\hline \multicolumn{6}{|l|}{ MMAS- $8^{\mathrm{b}}$} \\
\hline \multicolumn{6}{|l|}{ JP-ADDQoL ${ }^{c}$} \\
\hline \multicolumn{6}{|l|}{ All-cause death } \\
\hline \multicolumn{6}{|c|}{ Composite cardiovascular outcome } \\
\hline \multicolumn{6}{|l|}{ Composite renal endpoint } \\
\hline \multicolumn{6}{|c|}{ DialBetesPlus safety questionnaire } \\
\hline \multicolumn{6}{|l|}{ Safety } \\
\hline DialBetesPlus usage frequency & & & & & \\
\hline
\end{tabular}

${ }^{\mathrm{a} J-S D S C A}$ : Japanese version of the Summary of Diabetes Self-Care Activities Measure.

bMMAS-8: 8-item Morisky Adherence Scale.

${ }^{\mathrm{c}}$ JP-ADDQoL: Japanese version of the Audit of Diabetes-Dependent Quality of Life. 


\section{COVID-19 Related Adjustments}

Due to the COVID-19 pandemic, we made some adjustments to ensure complete data collection if participants canceled their hospital visits to avoid infection risk. The Research Ethics Committee of The University of Tokyo Graduate School of Medicine and its affiliated institutions formally approved adjustments to the trial protocol. First, we adopted a self-administered blood collection kit to measure blood glucose, $\mathrm{HbA}_{1 \mathrm{c}}$, eGFR, HDL-C, LDL-C, and TG levels when participants canceled in-person hospital visits. Albuminuria levels were also measured using the first-morning void mailed with the self-collected blood sample. We can conduct a blood examination by collecting $65 \mu \mathrm{l}$ of blood from a fingertip with the self-administered blood collection kit, Ouchide-doc (Halmek Ventures, Inc). It is reported that the assay results with this blood collection method are quite comparable to the conventional methods used in hospitals [33]. Second, to minimize the face-to-face contact for data collection, we sent questionnaires to participants' homes in advance of their hospital visits.

\section{Ethics and Dissemination}

The study is being carried out in compliance with the Declaration of Helsinki. This protocol and informed consent forms were approved by the Research Ethics Committee of The University of Tokyo Graduate School of Medicine and affiliated institutions. This study was registered in the University Hospital Medical Information Network Clinical Trials Registry (UMIN000033261) [34].

All participants are included after providing their signed and informed consent to participate in the trial. The participants are also informed of their right to withdraw from the study at any time. After the study concludes, data will be accessible by study groups for analysis and dissemination. All results of any analyses will be presented at major national and international scientific conferences and submitted for peer-reviewed journals of international repute and visibility.

\section{Statistical Analysis}

Data regarding patients' characteristics are presented as mean (SD) or median (IQR). We will compare changes in albuminuria levels, physical parameters, blood tests, and nutritional intake between the intervention and control groups. These will be analyzed using the 2-tailed $t$ test or Mann-Whitney U test, as appropriate. Changes in J-SDSCA, MMAS-8, and JP-ADDQoL scores will also be analyzed using the Mann-Whitney U test. We will compare the proportion of hypoglycemia during the study in the intervention group to the proportion in the control group using Fisher's exact test. $P$ values $<.05$ will be considered statistically significant. Statistical analyses will be performed using SAS (version 9.4; SAS Institute Inc).

\section{Results}

The study was approved in April 2018. We started recruiting patients in July 2018 and completed recruitment in August 2019. The final 72-week follow-up was completed in April 2021. The first results are expected to be available later in 2021.

We recruited 159 participants with written informed consent (Figure 1). We had 24 participants excluded due to withdrawal of consent $(21 / 159,13 \%)$ and ineligibility $(3 / 159,2 \%)$. No participants were excluded due to an inability to use DialBetesPlus. A participant in the control group and another in the intervention group were also excluded after randomization due to the late discovery of ineligibility. The baseline characteristics of the remaining 133 patients are shown in Multimedia Appendix 1. Data for continuous variables are expressed as mean (SD) or median (IQR).

Of the 150 participants, $3(2 \%)$ subsequently declined to continue and were excluded, resulting in 132 randomized participants. Table 2 displays the baseline demographic characteristics of the remaining 130 participants, excluding the 2 late-discovered ineligible participants. Data for continuous variables are expressed as mean (SD) or median (IQR). $P$ values for continuous variables were calculated with 2-tailed Student's $t$ test or Mann-Whitney's $U$ test. $P$ values for categorical variables were calculated with Fisher's exact test.

The baseline characteristics showed no significant differences between the control group and the intervention group. 
Table 2. Baseline patient characteristics.

\begin{tabular}{|c|c|c|c|c|}
\hline Characteristics & Total $(n=130)$ & Control $(n=61)$ & Intervention $(\mathrm{n}=69)$ & $P$ value \\
\hline Age (years), mean (SD) & $59.5(9.4)$ & $60.5(8.7)$ & $58.7(10.0)$ & .28 \\
\hline $\operatorname{Sex}, \mathbf{n}(\%)$ & & & & .55 \\
\hline Male & $96(73.8)$ & $47(77.0)$ & $49(71.0)$ & \\
\hline Female & $34(26.2)$ & $14(23.0)$ & $20(29.0)$ & \\
\hline \multicolumn{5}{|l|}{ Physical parameters, mean (SD) } \\
\hline BMI $\left(\mathrm{kg} / \mathrm{m}^{2}\right)$ & $28.5(4.6)$ & $28.3(4.0)$ & $28.6(5.2)$ & .67 \\
\hline Systolic BP ${ }^{\mathrm{a}}(\mathrm{mmHg})$ & $133.2(16.7)$ & $133.8(17.2)$ & $132.8(16.3)$ & .73 \\
\hline Diastolic BP (mmHg) & $82.0(10.7)$ & $83.2(10.2)$ & $80.9(11.0)$ & .23 \\
\hline Smoking status, n (\%) & & & & .79 \\
\hline Nonsmoker & $53(40.8)$ & $23(37.7)$ & $30(43.5)$ & \\
\hline Current smoker & $29(22.3)$ & $14(23.0)$ & $15(21.7)$ & \\
\hline Ex-smoker & $48(36.9)$ & $24(39.3)$ & $24(34.8)$ & \\
\hline Duration of diabetes (years), mean (SD) & $13.1(7.2)$ & $12.5(6.4)$ & $13.7(7.9)$ & .35 \\
\hline \multicolumn{5}{|l|}{ Laboratory test, median (Q1-Q3) } \\
\hline Fasting plasma glucose $(\mathrm{mg} / \mathrm{dL})$ & $144.0(124.0-174.0)$ & $139.0(124.0-159.0)$ & $150.0(123.0-185.0)$ & .37 \\
\hline $\mathrm{HbA}_{1 \mathrm{c}}^{\mathrm{b}}(\%)$ & $7.5(7.0-8.0)$ & $7.4(6.9-7.9)$ & $7.5(7.1-8.1)$ & .31 \\
\hline $\mathrm{LDL}^{\mathrm{c}}$ cholesterol $(\mathrm{mg} / \mathrm{dL})$ & $99.0(78.0-117.0)$ & $106.0(78.0-117.0)$ & $98.0(83.0-112.0)$ & .55 \\
\hline $\mathrm{HDL}^{\mathrm{d}}$ cholesterol $(\mathrm{mg} / \mathrm{dL})$ & $49.5(42.9-60.8)$ & $49.0(44.0-60.8)$ & $50.0(42.0-60.5)$ & .64 \\
\hline Triglycerides (mg/dl) & $155.5(100.0-261.0)$ & $149.0(100.0-261.0)$ & $158.0(100.0-252.0)$ & .64 \\
\hline Creatinine (mg/dL) & $0.78(0.65-0.92)$ & $0.82(0.68-0.93)$ & $0.78(0.62-0.92)$ & .33 \\
\hline $\mathrm{eGFR}^{\mathrm{e}}\left(\mathrm{mL} / \mathrm{min} / 1.73 \mathrm{~m}^{2}\right)$ & $72.0(61.8-85.3)$ & $71.0(63.8-83.0)$ & $76.5(59.5-85.5)$ & .29 \\
\hline $\mathrm{UACR}^{\mathrm{f}}(\mathrm{mg} / \mathrm{gCr})$ & $36.4(15.1-76.2)^{\mathrm{g}}$ & $32.3(14.8-70.6)$ & $41.0(15.1-78.0)$ & .49 \\
\hline
\end{tabular}

${ }^{\mathrm{a}} \mathrm{BP}$ : blood pressure.

${ }^{\mathrm{b}} \mathrm{HbA}_{1 \mathrm{c}}$ : glycated hemoglobin.

${ }^{\mathrm{c}}$ LDL-C: low-density lipoprotein cholesterol.

${ }^{\mathrm{d}}$ HDL-C: high-density lipoprotein cholesterol.

$\mathrm{e}_{\mathrm{eGFR}}$ : estimated glomerular filtration rate.

${ }^{\mathrm{f}} \mathrm{UACR}$ : urine albumin-to-creatinine ratio.

${ }^{\mathrm{g}}$ One case had a missing value.

\section{Discussion}

The beneficial effect of mHealth on T2DM in improving glycemic control has been widely reported $[14,35,36]$. However, the impact of mHealth on DKD, one of the major microvascular complications of T2DM, has not yet been shown. To our knowledge, this is the first study evaluating the efficacy of mHealth on DKD in which microalbuminuria is the primary endpoint and eGFR is one of the secondary endpoints. Furthermore, because we followed participants for 6 months after the intervention, this study enables us to assess if the novel smartphone-based self-management support system DialBetesPlus can discernibly modify self-care behaviors in T2DM patients.

DialBetesPlus is an improved version of the previously reported DialBetics [17]. The main upgrade is providing feedback on a patient's diet. The assessment is designed to provide positive feedback praising the patients' achievement, encouraging patients, and improving their self-efficacy. The system assesses a patient's diet precisely for each meal with an upgraded database. Additionally, patients can receive feedback on their daily and weekly diets to comprehensively look at their lifestyles.

While recent meta-analysis on mHealth shows that bidirectional communication between patients and health providers is indispensable for better glycemic control outcomes of T2DM patients [35,37], DialBetesPlus features a completely automated feedback system using the algorithm of DialBetics. Even though patients who used DialBetics cannot contact their health providers directly via DialBetics, a previous study showed significant reductions in $\mathrm{HbA}_{1 \mathrm{c}}(0.4 \%$ decrease), comparable 
to that achieved by other systems accompanied by interactive communication [17].

We hypothesize that albuminuria levels will significantly decrease in the intervention group compared to the control group due to improved self-care behaviors and glycemic control. This study may broaden the potential of mHealth to prevent the progression of T2DM microvascular complications.

\section{Acknowledgments}

The MMAS- 8 scoring and coding presented in the article were done using the electronic Morisky Widget MMAS- 8 Software. Use of the Morisky Widget MMAS-8 software, copyright registration number TX 8-816-517, is protected by US copyright laws. Permission for the use of the Morisky Widget MMAS-8 software is required and was obtained for this research. A license agreement is available from MMAS Research LLC 14725 NE 20th St Bellevue, WA 98007, USA; strubow@morisky.org.

The ADDQoL was applied in the study with the consent and license received from the author, Clare Bradley (Health Psychology Research Unit, Royal Holloway, University of London) [38]. Finally, we would like to thank the participants, physicians (see Multimedia Appendix 2), and other health care professionals at the 8 participating institutions. This research was supported by AMED (grant JP19ek0210095).

\section{Authors' Contributions}

KW, YT, KT, TY, TK, MN, NK, and KO contributed to the design and implementation of the study. YK, AS, KW, KM, AH, MM, HW, YT, SJ, NH, TY, SK, AI, SS, TM, UO, TI, and TT conceived and planned the study. YK, AS, and KW drafted the manuscript. $\mathrm{KW}$ is the principal investigator of the study and was responsible for conducting the study overall. All authors commented on the manuscript and approved the final version.

\section{Conflicts of Interest}

The author(s) disclose receipt of the external financial support for the research, authorship, and publication of this article: In addition to the AMED grant, this research was supported by NTT DOCOMO Inc. and Nihon Chouzai Co, Ltd. KM was a member of a cooperative program between the University of Tokyo and NTT DOCOMO.

\section{Multimedia Appendix 1}

Patient characteristics prior to randomization.

[DOCX File, 19 KB-Multimedia Appendix 1]

\section{Multimedia Appendix 2}

Physicians involved in participant recruitment.

[DOCX File, 19 KB-Multimedia Appendix 2]

\section{References}

1. IDF Diabetes Atlas, 8th Edition. Brussels: International Diabetes Federation; 2017.

2. Liyanage T, Ninomiya T, Jha V, Neal B, Patrice HM, Okpechi I, et al. Worldwide access to treatment for end-stage kidney disease: a systematic review. Lancet 2015 May 16;385(9981):1975-1982. [doi: 10.1016/S0140-6736(14)61601-9] [Medline: 25777665]

3. van der Velde M, Matsushita K, Coresh J, Astor BC, Woodward M, Levey A, Chronic Kidney Disease Prognosis Consortium, et al. Lower estimated glomerular filtration rate and higher albuminuria are associated with all-cause and cardiovascular mortality. A collaborative meta-analysis of high-risk population cohorts. Kidney Int 2011 Jun;79(12):1341-1352 [FREE Full text] [doi: 10.1038/ki.2010.536] [Medline: 21307840]

4. Persson F, Rossing P. Diagnosis of diabetic kidney disease: state of the art and future perspective. Kidney Int Suppl (2011) 2018 Jan;8(1):2-7 [FREE Full text] [doi: 10.1016/j.kisu.2017.10.003] [Medline: 30675433]

5. Viberti G, Wheeldon NM, MicroAlbuminuria Reduction With VALsartan (MARVAL) Study Investigators. Microalbuminuria reduction with valsartan in patients with type 2 diabetes mellitus: a blood pressure-independent effect. Circulation 2002 Aug 06;106(6):672-678. [doi: 10.1161/01.cir.0000024416.33113.0a] [Medline: 12163426 ]

6. Barnett AH, Bain SC, Bouter P, Karlberg B, Madsbad S, Jervell J, Diabetics Exposed to TelmisartanEnalapril Study Group. Angiotensin-receptor blockade versus converting-enzyme inhibition in type 2 diabetes and nephropathy. N Engl J Med 2004 Nov 04;351(19):1952-1961. [doi: 10.1056/NEJMoa042274] [Medline: 15516696]

7. Mann JFE, Ørsted DD, Brown-Frandsen K, Marso SP, Poulter NR, Rasmussen S, LEADER Steering CommitteeInvestigators. Liraglutide and Renal Outcomes in Type 2 Diabetes. N Engl J Med 2017 Aug 31;377(9):839-848. [doi: 10.1056/NEJMoa1616011] [Medline: 28854085] 
8. Neal B, Perkovic V, Mahaffey KW, de Zeeuw D, Fulcher G, Erondu N, CANVAS Program Collaborative Group. Canagliflozin and Cardiovascular and Renal Events in Type 2 Diabetes. N Engl J Med 2017 Aug 17;377(7):644-657. [doi: 10.1056/NEJMoa1611925] [Medline: 28605608]

9. Afkarian M, Zelnick LR, Hall YN, Heagerty PJ, Tuttle K, Weiss NS, et al. Clinical Manifestations of Kidney Disease Among US Adults With Diabetes, 1988-2014. JAMA 2016 Aug 09;316(6):602-610 [FREE Full text] [doi: 10.1001/jama.2016.10924] [Medline: 27532915]

10. Ogurtsova K, da RFJD, Huang Y, Linnenkamp U, Guariguata L, Cho NH, et al. IDF Diabetes Atlas: Global estimates for the prevalence of diabetes for 2015 and 2040. Diabetes Res Clin Pract 2017 Jun;128:40-50. [doi: 10.1016/j.diabres.2017.03.024] [Medline: 28437734]

11. Kitsiou S, Paré G, Jaana M, Gerber B. Effectiveness of mHealth interventions for patients with diabetes: An overview of systematic reviews. PLoS One 2017;12(3):e0173160 [FREE Full text] [doi: 10.1371/journal.pone.0173160] [Medline: 28249025]

12. Greenwood DA, Gee PM, Fatkin KJ, Peeples M. A Systematic Review of Reviews Evaluating Technology-Enabled Diabetes Self-Management Education and Support. J Diabetes Sci Technol 2017 Sep;11(5):1015-1027. [doi: 10.1177/1932296817713506] [Medline: 28560898]

13. Russell-Minda E, Jutai J, Speechley M, Bradley K, Chudyk A, Petrella R. Health technologies for monitoring and managing diabetes: a systematic review. J Diabetes Sci Technol 2009 Nov 01;3(6):1460-1471 [FREE Full text] [doi: 10.1177/193229680900300628] [Medline: 20144402]

14. Wu X, Guo X, Zhang Z. The Efficacy of Mobile Phone Apps for Lifestyle Modification in Diabetes: Systematic Review and Meta-Analysis. JMIR Mhealth Uhealth 2019 Jan 15;7(1):e12297 [FREE Full text] [doi: 10.2196/12297] [Medline: 30664494]

15. Alicic RZ, Rooney MT, Tuttle KR. Diabetic Kidney Disease: Challenges, Progress, and Possibilities. Clin J Am Soc Nephrol 2017 Dec 07;12(12):2032-2045 [FREE Full text] [doi: 10.2215/CJN.11491116] [Medline: 28522654]

16. Bullock A, Burrows NR, Narva AS, Sheff K, Hora I, Lekiachvili A, et al. Vital Signs: Decrease in Incidence of Diabetes-Related End-Stage Renal Disease among American Indians/Alaska Natives - United States, 1996-2013. MMWR Morb Mortal Wkly Rep 2017 Jan 13;66(1):26-32 [FRE Full text] [doi: 10.15585/mmwr.mm6601e1] [Medline: 28081061]

17. Waki K, Fujita H, Uchimura Y, Omae K, Aramaki E, Kato S, et al. DialBetics: A Novel Smartphone-based Self-management Support System for Type 2 Diabetes Patients. J Diabetes Sci Technol 2014 Mar 13;8(2):209-215 [FREE Full text] [doi: 10.1177/1932296814526495] [Medline: 24876569]

18. Waki K, Fujiu K, Hayashi A, Kimura S, Kobayashi H, Nangaku M, et al. DialBetics: Smartphone-Based Self-Management for Type 2 Diabetes Patients on Insulin Injections. J Diabetes Sci Technol 2016 Dec;10(3):804-805 [FREE Full text] [doi: 10.1177/1932296815619638] [Medline: 27460625]

19. Guide for the Treatment of Diabetes Mellitus in 2016. Tokyo: The Japan Diabetes Society; 2016.

20. Leehey DJ, Moinuddin I, Bast JP, Qureshi S, Jelinek CS, Cooper C, et al. Aerobic exercise in obese diabetic patients with chronic kidney disease: a randomized and controlled pilot study. Cardiovasc Diabetol 2009 Dec 09;8:62 [FREE Full text] [doi: 10.1186/1475-2840-8-62] [Medline: 20003224]

21. Straznicky NE, Grima MT, Lambert EA, Eikelis N, Dawood T, Lambert GW, et al. Exercise augments weight loss induced improvement in renal function in obese metabolic syndrome individuals. J Hypertens 2011 Mar;29(3):553-564. [doi: 10.1097/HJH.0b013e3283418875] [Medline: 21119532]

22. Guy M, Newall R, Borzomato J, Kalra PA, Price C. Diagnostic accuracy of the urinary albumin: creatinine ratio determined by the CLINITEK Microalbumin and DCA 2000+ for the rule-out of albuminuria in chronic kidney disease. Clin Chim Acta 2009 Jan;399(1-2):54-58. [doi: 10.1016/j.cca.2008.09.006] [Medline: 18834870]

23. Mogensen CE, Vestbo E, Poulsen PL, Christiansen C, Damsgaard EM, Eiskjaer H, et al. Microalbuminuria and potential confounders. A review and some observations on variability of urinary albumin excretion. Diabetes Care 1995 Apr;18(4):572-581. [doi: 10.2337/diacare.18.4.572] [Medline: 7497874]

24. Witte EC, Lambers Heerspink HJ, de Zeeuw D, Bakker SJL, de Jong PE, Gansevoort R. First morning voids are more reliable than spot urine samples to assess microalbuminuria. J Am Soc Nephrol 2009 Feb;20(2):436-443 [FREE Full text] [doi: 10.1681/ASN.2008030292] [Medline: 19092125]

25. Wang H, Li R, Liu R, Cui X, Pan W, Sun A. Second Morning ACR Could Be the Alternative to First Morning ACR to Assess Albuminuria in Elderly Population. J Clin Lab Anal 2016 Mar;30(2):175-179 [FREE Full text] [doi: 10.1002/jcla.21833] [Medline: 25589002]

26. Toobert DJ, Hampson SE, Glasgow RE. The summary of diabetes self-care activities measure: results from 7 studies and a revised scale. Diabetes Care 2000 Jul;23(7):943-950 [FREE Full text] [Medline: 10895844]

27. Morisky DE, Ang A, Krousel-Wood M, Ward HJ. Predictive validity of a medication adherence measure in an outpatient setting. J Clin Hypertens (Greenwich) 2008 May;10(5):348-354 [FREE Full text] [Medline: 18453793]

28. Krousel-Wood M, Islam T, Webber LS, Re RN, Morisky DE, Muntner P. New medication adherence scale versus pharmacy fill rates in seniors with hypertension. Am J Manag Care 2009 Jan;15(1):59-66 [FREE Full text] [Medline: 19146365] 
29. Morisky DE, DiMatteo MR. Improving the measurement of self-reported medication nonadherence: response to authors. J Clin Epidemiol 2011 Mar;64(3):255-7; discussion 258 [FREE Full text] [doi: 10.1016/j.jclinepi.2010.09.002] [Medline: 21144706]

30. Bradley C, Todd C, Gorton T, Symonds E, Martin A, Plowright R. The development of an individualized questionnaire measure of perceived impact of diabetes on quality of life: the ADDQoL. Qual Life Res 1999;8(1-2):79-91. [Medline: 10457741]

31. Ostini R, Dower J, Donald M. The Audit of Diabetes-Dependent Quality of Life 19 (ADDQoL): feasibility, reliability and validity in a population-based sample of Australian adults. Qual Life Res 2012 Oct;21(8):1471-1477. [doi: 10.1007/s11136-011-0043-0] [Medline: 22012024]

32. Hirose AS, Fujihara K, Miyamasu F, Iwakabe S, Shimpo M, Heianza Y, et al. Development and evaluation of the Japanese version of the Audit of Diabetes-Dependent Quality of Life for patients with diabetes. Diabetol Int 2016 Dec;7(4):384-390 [FREE Full text] [doi: 10.1007/s13340-016-0260-4] [Medline: $\underline{\text { 30603290] }}$

33. Shinya S, Masaru A, Akira H, Eisaku H, Susumu O. Development of an assay of seven biochemical items, HbA1c, and hematocrit using a small amount of blood collected from the fingertip. Clin Chim Acta 2012 Jan 18;413(1-2):192-197. [doi: 10.1016/j.cca.2011.09.021] [Medline: 21968067]

34. University Hospital Medical Information Network Clinical Trials Registry. URL: https://upload.umin.ac.jp/cgi-open-bin/ ctr/ctr view.cgi?recptno=R000037924 [accessed 2021-08-15]

35. Bonoto BC, de AVE, Godói IP, de LLLP, Godman B, Bennie M, et al. Efficacy of Mobile Apps to Support the Care of Patients With Diabetes Mellitus: A Systematic Review and Meta-Analysis of Randomized Controlled Trials. JMIR Mhealth Uhealth 2017 Mar 01;5(3):e4 [FREE Full text] [doi: 10.2196/mhealth.6309] [Medline: 28249834]

36. Cui M, Wu X, Mao J, Wang X, Nie M. T2DM Self-Management via Smartphone Applications: A Systematic Review and Meta-Analysis. PLoS One 2016;11(11):e0166718 [FREE Full text] [doi: 10.1371/journal.pone.0166718] [Medline: 27861583]

37. Garabedian LF, Ross-Degnan D, Wharam JF. Mobile Phone and Smartphone Technologies for Diabetes Care and Self-Management. Curr Diab Rep 2015 Dec;15(12):109. [doi: 10.1007/s11892-015-0680-8] [Medline: 26458380]

38. Health Psychology Research. URL: http://www.healthpsychologyresearch.com [accessed 2021-08-15]

\author{
Abbreviations \\ BP: blood pressure \\ DKD: diabetic kidney disease \\ eGFR: estimated glomerular filtration rate \\ ESRD: end-stage renal disease \\ $\mathbf{H b A}_{1 \mathbf{c}}$ : hemoglobin $\mathrm{A}_{1 \mathrm{c}}$ \\ HDL-C: high-density lipoprotein cholesterol \\ JDS: Japan Diabetes Society \\ JP-ADDQoL: Japanese version of the Audit of Diabetes-Dependent Quality of Life \\ LDL-C: low-density lipoprotein cholesterol \\ mHealth: mobile health \\ MMAS-8: 8-item Morisky Adherence Scale \\ NFC: near field communication \\ QoL: quality of life \\ J-SDSCA: Japanese version of the Summary of Diabetes Self-Care Activities Measure \\ TG: triglycerides \\ T2DM: type 2 diabetes mellitus
}

Edited by $G$ Eysenbach; submitted 08.06.21; peer-reviewed by $R$ Khan; comments to author 29.06.21; revised version received 05.07.21; accepted 05.07.21; published 17.08.21

Please cite as:

Kawai Y, Sankoda A, Waki K, Miyake K, Hayashi A, Mieno M, Wakui H, Tsurutani Y, Saito J, Hirawa N, Yamakawa T, Komiya S, Isogawa A, Satoh S, Minami T, Osada U, Iwamoto T, Takano T, Terauchi Y, Tamura K, Yamauchi T, Kadowaki T, Nangaku M, Kashihara $N$, Ohe $K$

Efficacy of the Self-management Support System DialBetesPlus for Diabetic Kidney Disease: Protocol for a Randomized Controlled Trial

JMIR Res Protoc 2021;10(8):e31061

URL: https://www.researchprotocols.org/2021/8/e31061

doi: $10.2196 / 31061$

PMID: 
(C) Yuki Kawai, Akiko Sankoda, Kayo Waki, Kana Miyake, Aki Hayashi, Makiko Mieno, Hiromichi Wakui, Yuya Tsurutani, Jun Saito, Nobuhito Hirawa, Tadashi Yamakawa, Shiro Komiya, Akihiro Isogawa, Shinobu Satoh, Taichi Minami, Uru Osada, Tamio Iwamoto, Tatsuro Takano, Yasuo Terauchi, Kouichi Tamura, Toshimasa Yamauchi, Takashi Kadowaki, Masaomi Nangaku, Naoki Kashihara, Kazuhiko Ohe. Originally published in JMIR Research Protocols (https://www.researchprotocols.org), 17.08.2021. This is an open-access article distributed under the terms of the Creative Commons Attribution License (https://creativecommons.org/licenses/by/4.0/), which permits unrestricted use, distribution, and reproduction in any medium, provided the original work, first published in JMIR Research Protocols, is properly cited. The complete bibliographic information, a link to the original publication on https://www.researchprotocols.org, as well as this copyright and license information must be included. 